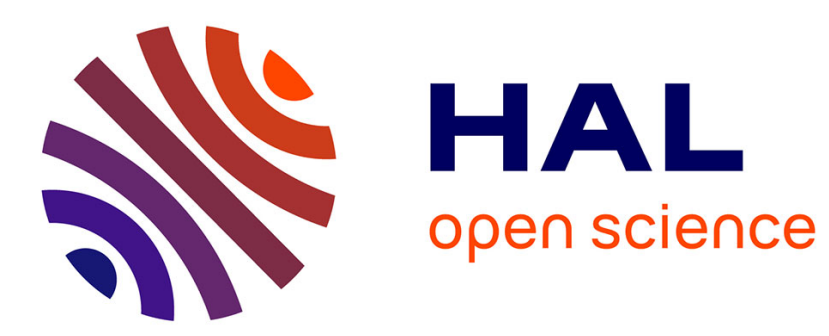

\title{
Générateur de fonction à commutation
}

B. Chocat, B. Eude, F. Lechevrel

\section{To cite this version:}

B. Chocat, B. Eude, F. Lechevrel. Générateur de fonction à commutation. Revue de Physique Appliquée, 1974, 9 (5), pp.901-906. 10.1051/rphysap:0197400905090100 . jpa-00243855

\section{HAL Id: jpa-00243855 https://hal.science/jpa-00243855}

Submitted on 1 Jan 1974

HAL is a multi-disciplinary open access archive for the deposit and dissemination of scientific research documents, whether they are published or not. The documents may come from teaching and research institutions in France or abroad, or from public or private research centers.
L'archive ouverte pluridisciplinaire HAL, est destinée au dépôt et à la diffusion de documents scientifiques de niveau recherche, publiés ou non, émanant des établissements d'enseignement et de recherche français ou étrangers, des laboratoires publics ou privés. 


\title{
GÉNÉRATEUR DE FONCTION A COMMUTATION
}

\author{
B. CHOCAT, B. EUDE et F. LECHEVREL
}

Laboratoire de Recherche Appliquée de l'Ecole Nationale Supérieure d'Electronique et d'Electromécanique de Caen, 14032 Caen Cedex, France

(Reçu le 28 janvier 1974)

Résumé. - Nous décrivons un générateur de fonction électronique à commutation qui simule la caractéristique invariante d'un système physique quelconque. Ce générateur dont nous exposons le principe, le mode de fonctionnement et les avantages doit remplacer, dans les calculateurs analogiques et dans les chaînes de mesure ou de commande, les générateurs classiques à diodes dont les performances et les facilités d'emploi sont devenues insuffisantes.

\begin{abstract}
We describe a switching function generator which simulates the time-invariant characteristic of any physical system. We set forth the principle, the directions for use and the advantages of this generator, which is to take the place of the conventional diode generator in analog computers and measurement or control systems.
\end{abstract}

1. Introduction. - Les générateurs de fonction (GF) utilisés tant au laboratoire (simulation, calcul analogique) que dans des processus industriels (chaîne de mesure ou de commande) sont des dispositifs, généralement électroniques, qui, soumis à une tension $V_{\mathrm{e}}$, délivrent une tension $V_{\mathrm{s}}=f\left(V_{\mathrm{e}}\right)$; la fonction $f$, connue a priori par un tableau valeur, est représentée (Fig. 1) par une caractéristique $(\Gamma)$.

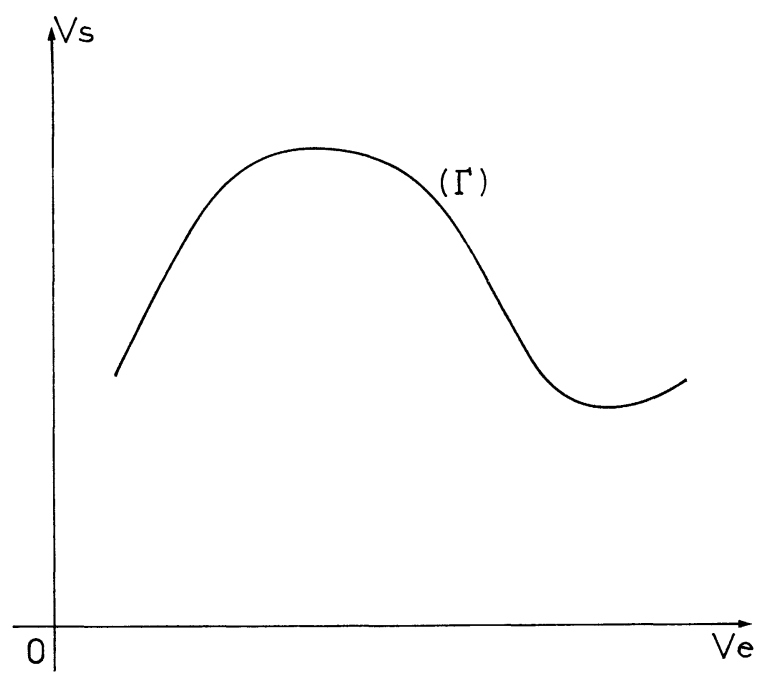

FIG. 1. - Caractéristique $(\Gamma)$ temporellement invariante $V_{\mathrm{s}}=f\left(V_{\mathrm{e}}\right)$.

De nombreuses études leur ont été consacrées ces dernières années [1 à 48]. Actuellement, on utilise les générateurs à diodes associés ou non à un rotateursymétriseur [20]. Pour pallier leurs défauts (voir par exemple [18]), nous avons mis au point un générateur de conception originale.

2. Principe du générateur. Description et fonctionnement. - Le générateur comporte deux modules (Fig. 2) :

- un module de calcul (analogique),

- un module de commande (séquentiel).

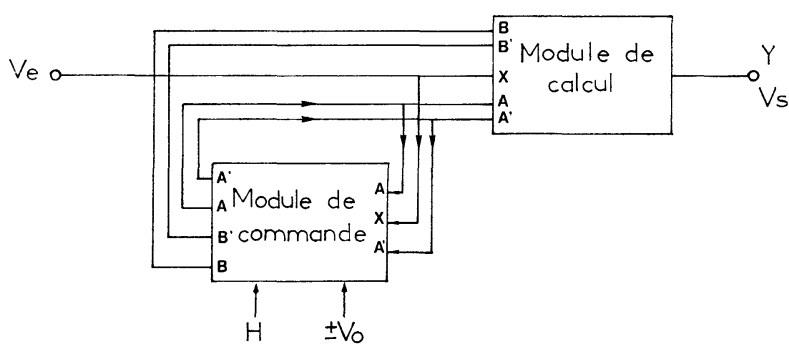

Fig. 2. - Schéma synoptique du générateur à commutation.

2.1 Module De Calcul. - 2.1.1 Principe - Ce module définit une approximation par segments de droites d'une caractéristique $(\Gamma)$ par le calcul préalable de la pente du segment $M_{i} M_{i-1}$ (Fig. 3) à l'aide des coordonnées $\left(x_{i}, y_{i}\right)$ et $\left(x_{i-1}, y_{i-1}\right)$ de deux points $M_{i}$ et $M_{i-1}$ appartenant à $(\Gamma)$. Ces points sont appelés points de commutation. 


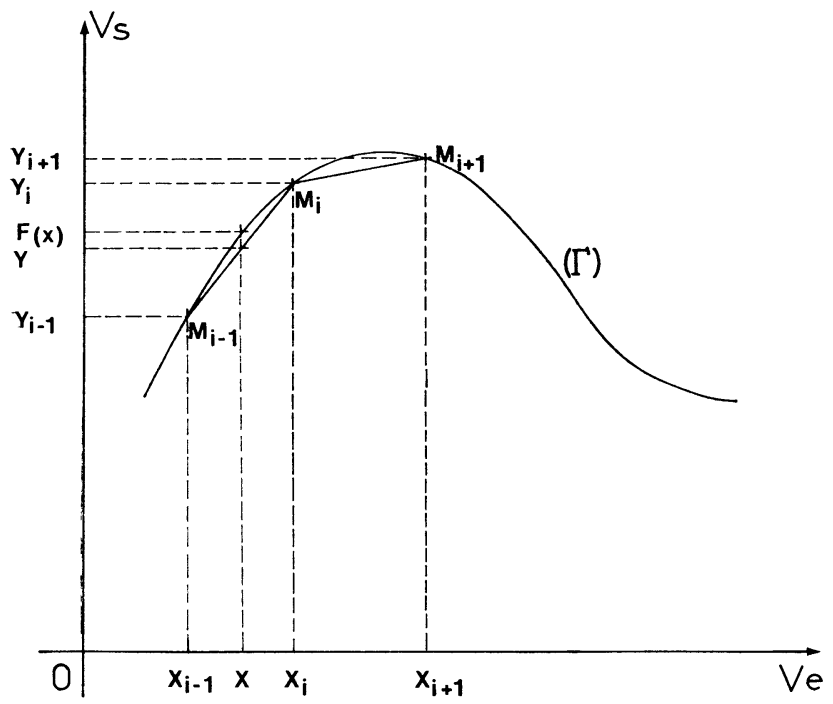

Fig. 3. - Distribution des points de commutation $\boldsymbol{M}_{\boldsymbol{i}}$ sur la caractéristique $(\Gamma)$.

Soit $x$ la valeur de la tension d'entrée si $x_{i-1}<x<x_{i}$

$$
\begin{aligned}
y & =y_{i-1}+\frac{y_{i}-y_{i-1}}{x_{i}-x_{i-1}}\left(x-x_{i-1}\right) \\
& =y_{i-1}+\frac{\Delta_{y i}}{\Delta_{x i}}\left(x-x_{i-1}\right)
\end{aligned}
$$

$y$ est la valeur de la tension de sortie du module.

La distribution des points $M_{i}\left(x_{i}, y_{i}\right)$ (nombre et densité) détermine la précision de l'approximation. $\mathrm{Si}\left|\varepsilon_{i}\right|_{\max }=|y-f(x)|_{\max }, x_{i-1}<x<x_{i}$, nous pouvons qualifier la précision par $\operatorname{Sup}\left|\varepsilon_{i}\right|_{\max }, \forall_{i}(i=0$ à $n$ ). La définition de la caractéristique $(\Gamma)$ par segments de droite sera d'autant meilleure que sup $\left|\varepsilon_{i}\right|_{\max }$ sera faible ; pour un nombre $n+1$ points $M_{i}$ donné, il suffira de choisir les points de telle sorte que

$$
\left|\varepsilon_{0}\right|_{\max }=\left|\varepsilon_{1}\right|_{\max }=\cdots=\left|\varepsilon_{i}\right|_{\max }=\ldots=\left|\varepsilon_{n}\right|_{\max } .
$$

La solution la plus efficace est alors de tracer cette caractéristique $(\Gamma)$ et de placer les points $M_{i}$ répondant à cette condition. Il apparaît qu'il peut être intéressant dans cette optique de choisir des points $M_{i}$ n'appartenant pas à $(\Gamma)$ (Fig. 4).

2.1.2 Schéma de principe. - Dans ce schéma (Fig. 5) $\alpha_{i}$ et $\beta_{i}$ sont des potentiomètres qui, alimentés par une tension de référence $\left( \pm V_{0}\right)$, introduisent les valeurs numériques, contrôlées par voltmètre en $\mathrm{A}, \mathrm{A}^{\prime}$, B, B', $x_{i}, x_{i-1}, y_{i}, y_{i-1}$, dans les soustracteurs $S_{x i}, S_{x}$, $S_{y i}$. Le multiplieur $M$, le diviseur $D$ et le sommateur $S$ calculent $y$. Pour les valeurs $x$ de la tension d'entrée non comprises dans l'intervalle $\left(x_{i-1}, x_{i}\right)$, un bloc de commande commute les entrées $\mathrm{A}, \mathrm{A}^{\prime}, \mathbf{B}, \mathrm{B}^{\prime}$ sur les potentiomètres adéquats. Par exemple: $x_{i}<x<x_{i+1}$, $\mathrm{A}, \mathrm{A}^{\prime}, \mathrm{B}, \mathrm{B}^{\prime}$ sont reliés respectivement aux potentiomètres $\alpha_{i+1}, \alpha_{i}, \beta_{i+1}, \beta_{i}$ préalablement réglés aux valeurs $x_{i+1}, x_{i}, y_{i+1}, y_{i}$.

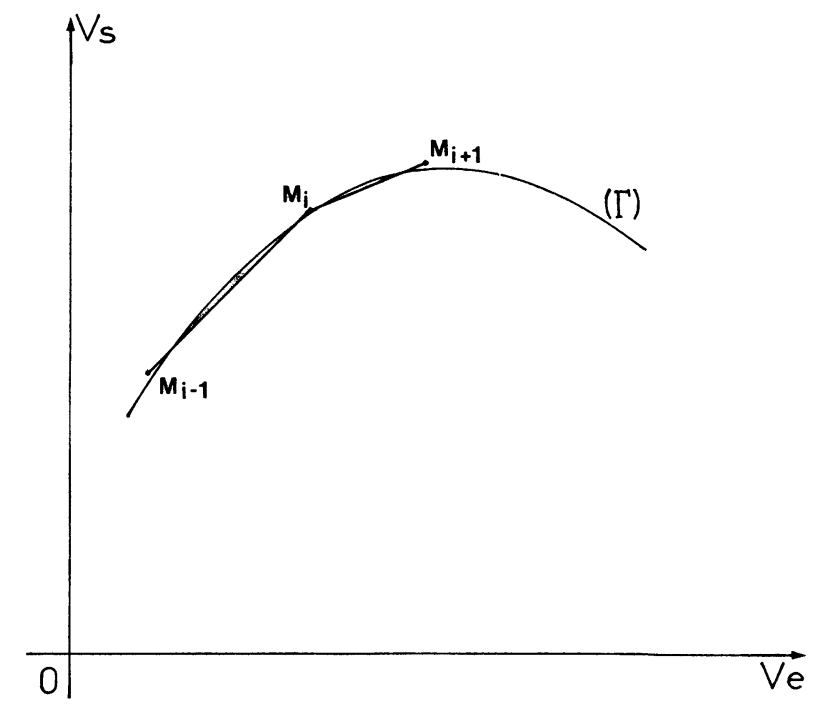

FIG. 4. - Choix de points de commutation $M_{i}$ à précision maximum.

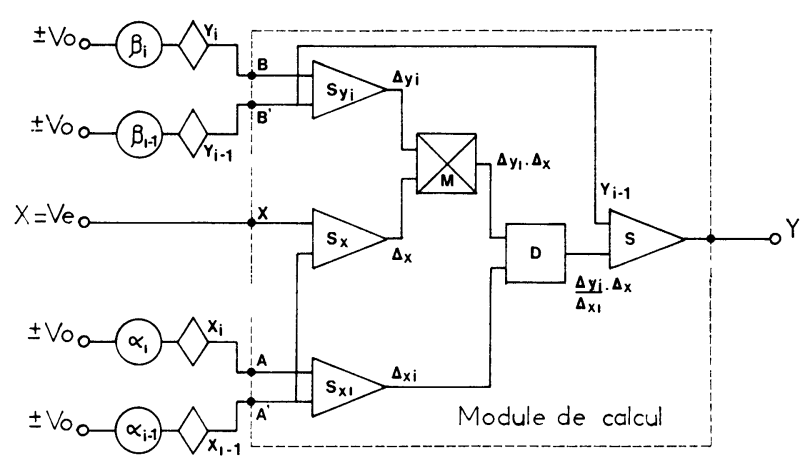

Fig. 5. - Module de calcul.

2.2 Module de commande. - Le bloc de commande (Fig. 6) a pour but de commuter les entrées $\mathrm{AA}^{\prime}$ $\mathrm{BB}^{\prime}$ aux potentiomètres $\alpha$ et $\beta$ correspondant à la plage $\left(x_{i-1}, x_{i}\right)$ de la tension $x$ lorsque $x_{i-1}<x<x_{i}$.

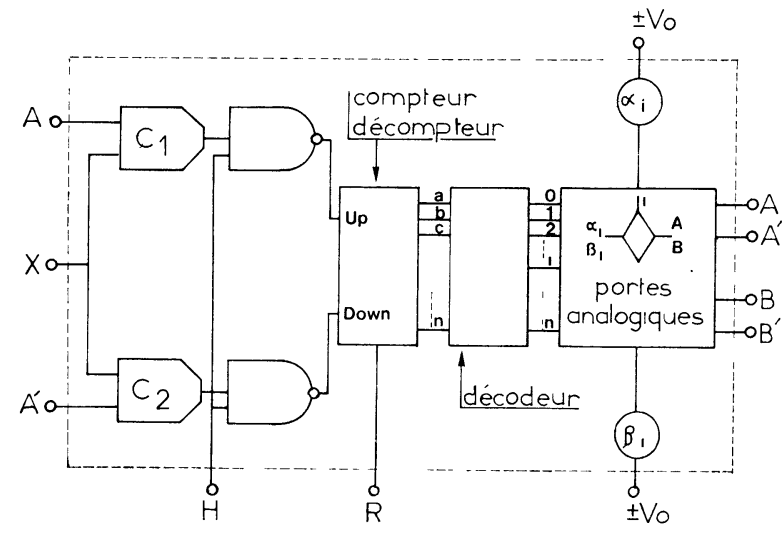

FIG. 6. - Module commande. 
Il est constitué de :

- deux comparateurs $C_{1}$ et $C_{2}$ tels que si $V_{\mathrm{A}}$ et $V_{\mathrm{A}^{\prime}}$ sont les tensions d'entrée en $\mathrm{A}$ et $\mathrm{A}^{\prime}$

$$
\begin{array}{ll}
x>V_{\mathrm{A}}: C_{1}=1 & x>V_{\mathrm{A}^{\prime}}: C_{2}=0 \\
x<V_{\mathrm{A}}: C_{1}=0 & x<V_{\mathrm{A}^{\prime}}: C_{2}=1
\end{array}
$$

- d'un compteur-décompteur (à $2^{N}$ ) qui :

- compte (ou décompte) sur les fronts descendants des signaux appliqués en $U$ (ou D), l'entrée $\mathrm{D}(\mathrm{ou} \mathrm{U})$ étant à un niveau logique haut ;

- reste en position si les entrées $U$ et $D$ sont simultanément à de niveaux hauts ;

- d'un bloc de décodage à $n$ sorties,

- de $4 n$ portes analogiques reliant les potentiomètres $\alpha$ et $\beta$ aux entrées $\mathrm{AA}^{\prime} \mathbf{B B}^{\prime}$ du module de calcul (Fig. 7).

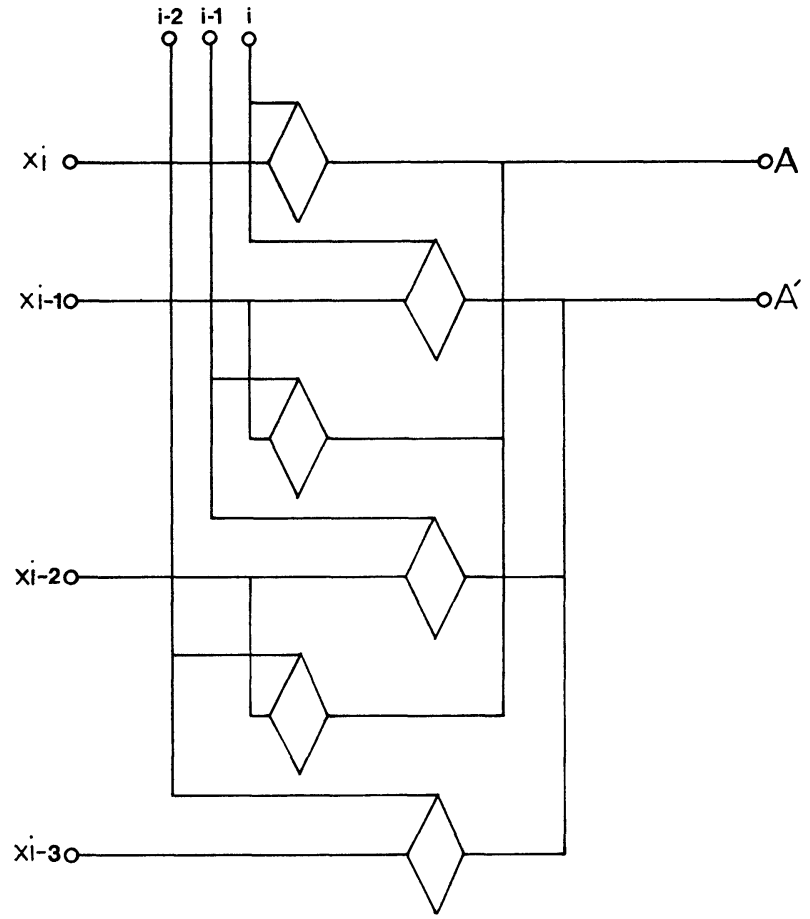

Fig. 7. - Commande des portes analogiques.

Un générateur d'impulsions, l'horloge $\mathrm{H}$, pilote le module; le bloc de commande fonctionne ainsi : soit un état initial $V_{\mathrm{e}}=x\left(x_{i-1}<x<x_{i}\right)$.

L'état du compteur est tel que les potentiomètres $\alpha_{i}$, $\alpha_{i-1}, \beta_{i}, \beta_{i-1}$ sont reliés aux entrées $\mathrm{A}, \mathrm{A}^{\prime}, \mathrm{B}$ et $\mathbf{B}^{\prime}$. Les entrées des comparateurs $C_{1}$ et $C_{2}$ sont soumises aux tensions $V_{\mathrm{A}}=x_{i}$ et $V_{\mathrm{A}^{\prime}}=x_{i-1}, C_{1}=C_{2}=0$. Le module de commande est dans un état stable.

Soit une tension $V_{\mathrm{e}}=x^{\prime} \neq x$.

Si $x_{i-1}<x^{\prime}<x_{i}$, les sorties logiques des comparateurs ne changent pas et le module de commande reste dans l'état.

Si $x^{\prime}>x_{i}, C_{1}=1$ le compteur est en position de comptage ; les entrées $\mathrm{A}$ et $\mathrm{A}^{\prime}$ des comparateurs sont portées, à chaque impulsion, successivement aux tensions $\left(x_{i+1}, x_{i}\right),\left(x_{i+2}, x_{i+1}\right), \ldots$, etc., le comptage s'arrête $C_{1}=0$ à la suite de $p$ impulsions si

$$
x_{i+p-1}<x^{\prime}<x_{i+p}
$$

(Fig. 8).

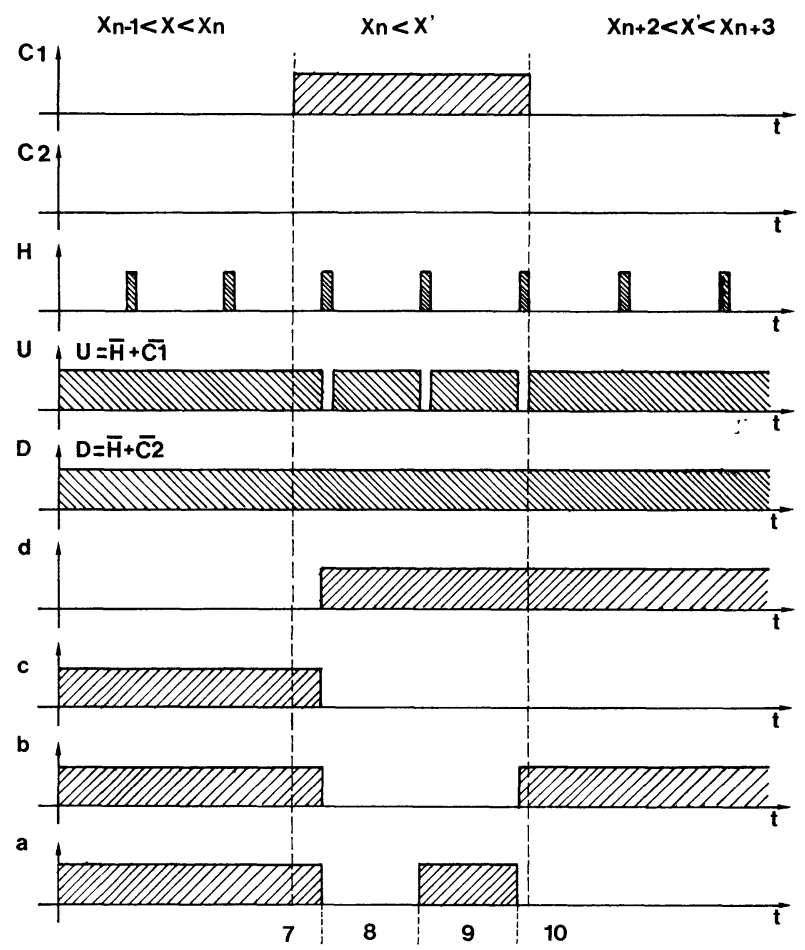

FIG. 8. - Diagramme logique temporel des commutations.

Si $x<x_{i-1}, C_{2}=1$ même processus mais dans le sens du décomptage.

Le module de commande est un système bouclé piloté par horloge ; pour obtenir un état stable, il est nécessaire et suffisant que $x_{\mathrm{m}}<x<x_{\mathrm{M}}$ avec $x_{\mathrm{m}}=\operatorname{Min}\left(x_{i}\right), x_{\mathrm{M}}=\operatorname{Max}\left(x_{i}\right) \forall_{i}(i=0$ à $n)$. En effet, si $x>x_{M}$ (ou $\left.x<x_{M}\right)$, le comparateur $C_{1}$ (ou $C_{2}$ ) est à 1 en permanence et le compteur compte (ou décompte) sans s'arrêter à un état stable. Pour plus de sécurité, à la construction, le compteur est bloqué à zéro lorsqu'on décompte et bloque à $2^{N}$ lorsque l'on compte. Le réglage du générateur est ainsi effectué (Fig. 9) :

$x_{0}=x_{\mathrm{m}} \quad \forall x<x_{1}, \quad$ le compteur est à $0 \quad$ (Fig. 9a) $x_{n}=x_{\mathrm{M}} \quad \forall x_{n-1}<x$, le compteur est à $2^{N}$ (Fig. 9b).

3. Mise en œuvre. - La mise en œuvre du générateur est très rapide ; disposant d'un tableau de valeurs $x_{i}, y_{i}$, il suffit, pour régler le générateur, de porter au voltmètre les sorties des potentiomètres $\alpha_{i}$ et $\beta_{i}$, dans un ordre quelconque ; les réglages des potentiomètres sont indépendants. On peut dire dès lors que la rapidité de l'opération retire tout intérêt aux atténuateurs numériques. On sait, en effet, que, la mise en œuvre des générateurs de fonctions à diodes étant longue et fastidieuse, 


\section{Dépassement}

\section{$\forall \mathrm{x}: \mathrm{x}<\mathrm{x}_{1}$}

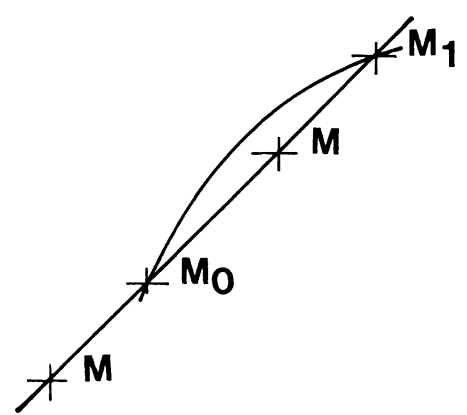

(a)

\section{$\forall \mathrm{x}: \mathrm{x}_{\mathrm{n}-1}<\mathrm{x}$}

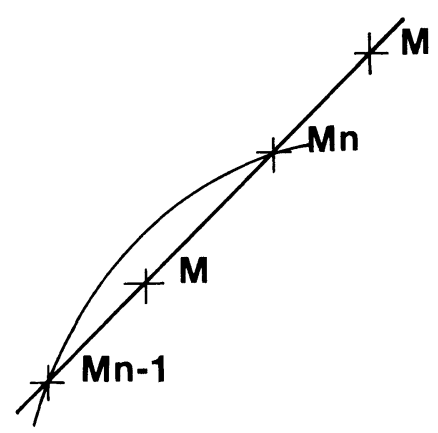

(b)

FIG. 9. - Fonctionnement du générateur à l'extérieur de sa plage normale de travail. a) $\mathrm{M}(x y)$ décrit la demi-droite $\mathrm{M}_{1}, \mathrm{M}_{0}$; b) $\mathrm{M}(x y)$ décrit la demi-droite $\mathrm{Mn}-1, \mathrm{Mn}$.

on eut l'idée de remplacer chaque potentiomètre par un atténuateur numérique. Il suffit, alors, d'utiliser un jeu de cartes, correspondant à la caractéristique $(\Gamma)$ désirée, pour régler les atténuateurs aux valeurs souhaitées. Malheureusement cette solution - adaptable dans ce générateur à commutations - est très onéreuse ; de plus, elle ne tient pas compte de dérives inévitables des opérateurs analogiques. Pour améliorer la précision de réglage, nous étudions une modification du module de commande permettant de bloquer, à la demande, le compteur à l'état $i$; ainsi affichant la valeur $x=x_{i}$ à l'entrée du module de calcul, le potentiomètre $\beta_{i}$ sera réglé de sorte qu'à la sortie de ce module, $y$ prenne la valeur $y_{i}$.

4. Performances. Avantages et inconvénients. Avec le matériel utilisé, l'expérience a montré que, jusqu'à une fréquence de balayage de $100 \mathrm{~Hz}$ pour la tension d'entrée, la caractéristique obtenue recouvre à $2 \%$ près la caractéristique à segments prévue. Cette précision de calcul est fortement tributaire de la pré- cision du voltmètre numérique d'étalonnage et du réglage des amplificateurs du module calcul. Il serait possible de travailler à une fréquence plus élevée à la condition d'utiliser des opérateurs logiques ou analogiques plus rapides et d'introduire des retards ajustables dans les chaînes directes du module calcul (élimination des aléas de commutation).

4.1 Les avantages du dispositif sont appréciables; il est possible :

a) de simuler toute caractéristique établissant une correspondance biunivoque entre deux grandeurs;

b) de répartir au mieux les points $M_{i}$ sur la caractéristique $(\Gamma)$ à simuler de sorte que la précision de définition soit maximum pour un nombre de points de commutation donné ;

c) d'obtenir (Fig. 10) des caractéristiques de pente très grande, pratiquement infinie (au moins 100 c'est-àdire $100 \mathrm{mV}-10 \mathrm{~V}$ ) ;

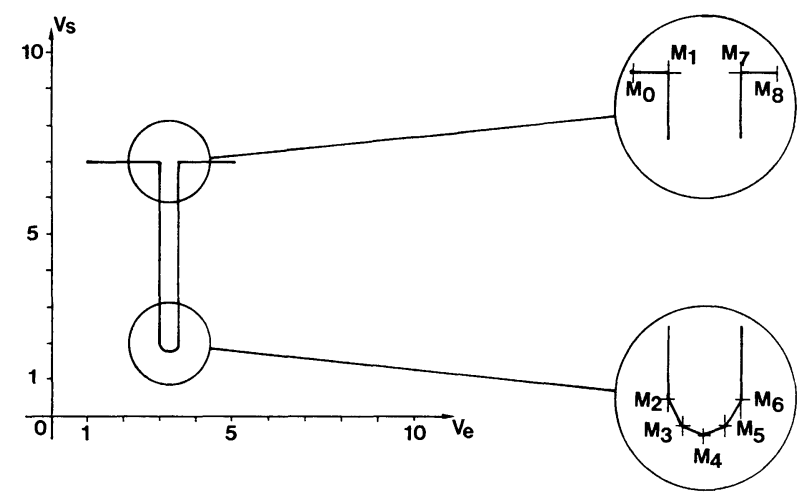

Fig. 10. - Exemple de caractéristique réalisable sur le générateur à commutation.

d) de simuler des caractéristiques non biunivoques. Ainsi figure 11, peut-on, sans montage extérieur, simuler un cycle d'hystérésis, quelconque, en particulier non symétrique.

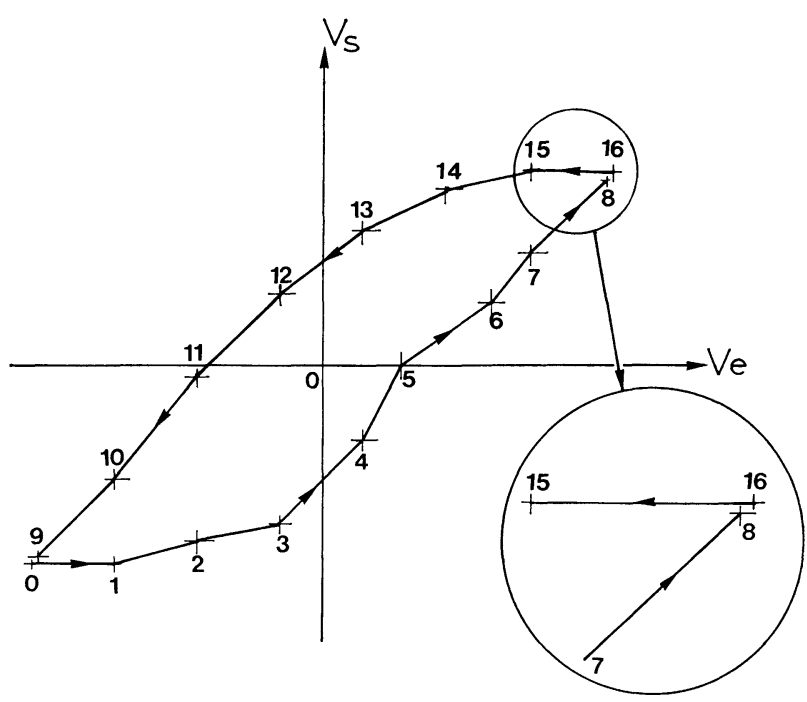

Fig. 11. - Cycle d'hystérésis. 
En régime établi, le cycle est décrit suivant les flèches; sur la branche supérieure le compteur décompte, sur la branche inférieure le compteur compte. Pratiquement, au réglage du générateur, on peut confondre les coordonnées des points $(16,8)(0,9)$. Le passage du segment $(7,8)$ au segment $(15,16)$ découle de l'exploration séquentielle des états du module de commande jusqu'à un état stable caractérisé par $C_{1}=C_{2}=0$. Il suffit que la tension d'entrée dépasse légèrement $x_{8}$ pour lancer le compteur en comptage ;

$e)$ d'associer enfin plusieurs générateurs de ce type pour engendrer des fonctions de fonctions ou pour augmenter la précision de définition d'une caractéristique (cf. Fig. 12).
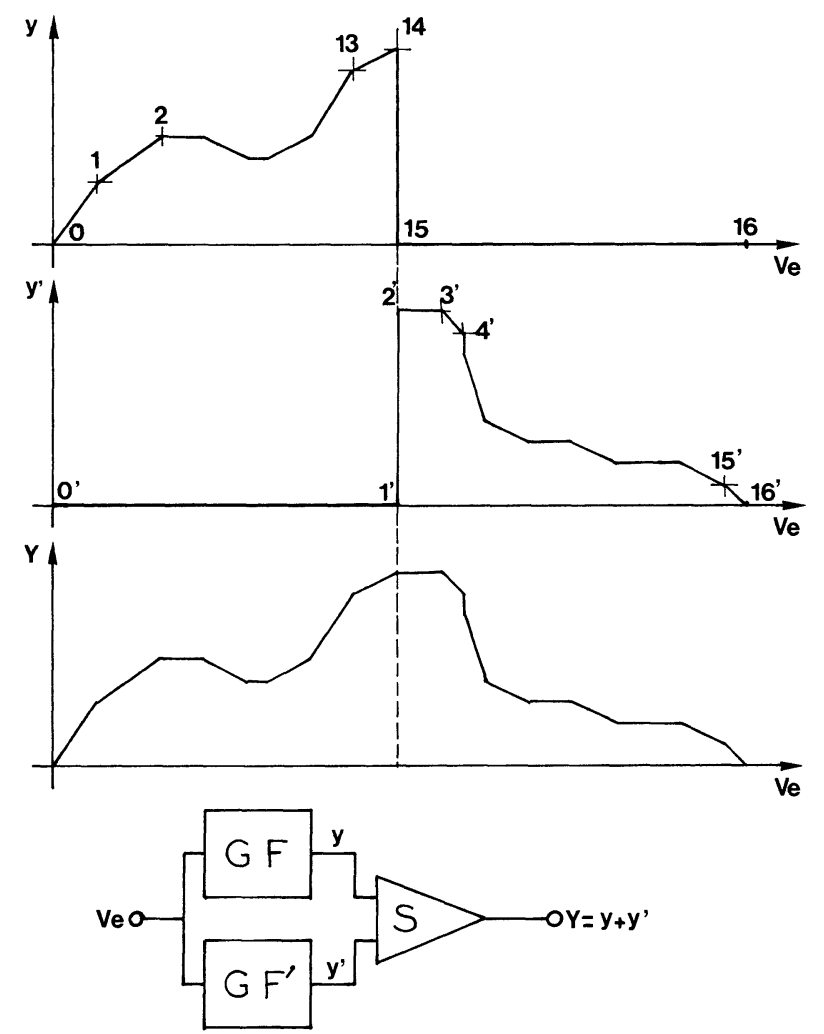

FIG. 12. - Association particulière de deux générateurs à commutation.

4.2 Les inconvénients propres au montage sont de deux ordres :

a) Lors du passage à un point de commutation, on observe un saut de la tension de sortie (Fig. 13).

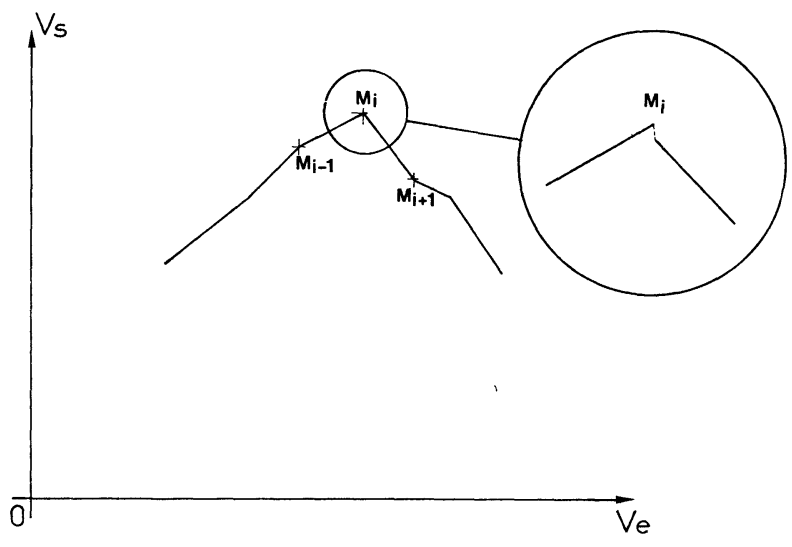

Fig. 13. - Saut de la tension de sortie à la commutation.

Ce saut limite, d'une part, la précision du résultat mais, d'autre part, risque d'engendrer des perturbations parasitaires dans un système rapide placé en aval du générateur. Cet inconvénient est pratiquement éliminé par un réglage soigné du module de calcul, par un choix de multiplieurs de qualité et par un échantillonnage-blocage de la tension de sortie à la commutation.

b) Contrairement aux générateurs à diodes dont les caractéristiques ne sont pas parfaitement linéaires, le générateur délivre, dans chaque intervalle $\left(x_{i-1}, x_{i}\right)$, des segments de droites; il en résulte des cassures aux points de commutation.

5. Application. Développements. - Les applications d'un tel dispositif sont bien entendu celles classiques d'un générateur de fonctions à diodes avec des commodités plus grandes et un domaine d'emploi plus large. En particulier, nous pensons qu'un tel générateur pourrait s'insérer dans des chaînes de mesure ou de commande, par exemple pour linéariser la caractéristique d'un capteur, ou pour dilater une échelle.

Dans le premier cas, mis en série avec un capteur, le générateur préalablement ajusté délivre un signal « quasi proportionnel » à la grandeur d'entrée du capteur.

Dans le second, le générateur joue le rôle d'amplificateur à gain variable dans une ou plusieurs zones de variation d'une grandeur que l'on souhaite particulièrement surveiller, en quelque sorte c'est une loupe.

\section{Bibliographie}

\section{Publications.}

[1] Balaboshro, Maslov, Sakhrov, Méthode de synthèse sur ordinateur d'un générateur de fonction à diodes. Avtomatika i telemekhanica S. S. S. R. (1972) p. 135/141 (5 réf.).

[2] BeLLo, Design of a diode function-generator using the diode equation and iteration. IEEE circuit theory USA 19 (1972) $213 / 214$ (7 ref.).
[3] - An improved method for the design of electronic function generator network. Meas. and control GB 5 (1972) 271-5.

[4] Dang Phnocly, Nguyen Tan Hao, La magnétorésistance dans la génération analogique de fonctions polynominales. C. R. Hebd. Séan. Acad. Sci. 272 (1971) 486-9.

[5] IL NICKIJ, BolBOT, Générateur de fonctions réversible reproduisant des fonctions rationnelles. Izv. Vyssh. Ucheb. Zaved. Proborostr. S. S. S. R. 14 (1971) 65/67 (3 réf.). 
[6] Rodriguez, IzQuierdo, Civit, A., High gain integrated amplifiers stabilize function generators. An. Ass. Int. Calcul analogique (Belgique) 13 (1971) 156/159.

[7] Burren, R. S., Linearize almost anything with multipliers. Electron. Des. USA 19 (1971) 74/75.

[8] ROBERTSON, Digitally controlled analog function generator (1971) PAT USA 3557347.

[9] Yans, Approximations linéaires en vue de la conception optimale des GF à diodes. Bull. Sci. AIM (B) 84 (1971) 191-206 (10 réf.).

[10] Fisher, DraXler, Réalisation de générateurs de fonction non linéaires. Nachr-Tech Z Dtsch 23 (1970) 262-264 (12 réf.).

[11] Liversidge, M. H., Low-cost reciprocal law circuit. Electronic Letters (GB) 6 (1970) 389-390 (5 réf.).

[12] GRIMBLEBY, Low high performance reciprocal law circuit. Electronic letters $(G B) 6$ (1970) 755-756 (2 réf.).

[13] Morris, Saturating operational amplifiers add up to a simple way to compress AC signals over many decades. Electronics USA 43 (1970) 105-107.

[14] Dikis, Efimov, Poralfaer, StanishevskiJ, Générateur de fonctions délivrant les accroissements de fonction d'après les accroissements donnés de l'argument. Izv. Vyssh. Ucheb. Zaved. Probovostr S. S. S. R. 13 (1970) 48-51 (3 réf.).

[15] BARNES, A function generator using hybrid techniques. Radio Electronic Engr. GB 39 (1970) 153-8 (3 réf.).

[16] RADEKA, Rogers, Fast polynominal function generator. Rev. Sci. Instrum. USA 41 (1970) 498-503 (3 réf.).

[17] Gaghano, Googe, NeIL, Hybrid function generator. IEEE Trans. Inst. Meas. USA 18 (1969) 110-3.

[18] Cate, Designing with non-linear function modules. Elect. Equip. Engng. Mag. Circuit. Engng USA 17 (1969) 62-71 (7 réf.).

[19] Molchanov, Etude de la transformation fonctionnelle de grandeurs électriques à l'aide du spectrotrons (circuit résonnant non linéaire). Avtomatikha i telemechanika S. S. S. R. (1969) p. 160-8 (5 réf.).

[20] Fravalo, Contribution à l'étude des quadripôles rotateurs et symétriseurs. Application à la réalisation d'un générateur de fonction. Thèse de $3^{\mathrm{e}}$ cycle Rennes 1969 (18 réf.).

[21] Function generator, Westing. Elec. Corp. Pat UK 1245612 (1968).

[22] SchleIfER, Générateur de fonction à diodes des fonctions empiriques. Int. Elekt. Rdsch. Dtsch 21 (1967) 235-7 ( 2 réf.).

[23] Frankel, Convertisseurs fonctionnels avec modulateur de Hall. Electrotehnica Româna 14 (1968) 288-93.

[24] LECHENU, Générateur de fonction à potentiomètre. Electronique industrielle (1961) p. 299-302.
[25] NoIKA, Generating tangental sweep for infrared mapping. Electronics USA 34 (1961) 64-66.

[26] Galli, Non ideal diodes and practical generators. Cont. Engng. USA 7 (1960) 107-9.

[27] Gilbert, The design of position and velocity servos for multiplying and function-generators. IRE Trans. Elec. computer USA 8 (1959) 391-9.

[28] MiURA Abe, Error analysis of a photo former. J. Japan 5 (1959) 3-7.

[29] Young Alexander Schwetetmann, Rotating-dish function generator for analog computer. Rev. Sci. Instrum. USA 30 (1959) 318-22.

[30] Tomovic, Contribution à la réalisation pratique des opérateurs universels non linéaires, $2^{\mathrm{es}}$ journées Int. Calcul analogique Strasbourg 1958 (Masson) 29 (1959) $5 \times 21$, p. 120-7.

\section{OUVRAGES.}

[31] Analog computation. Jackson (McGraw Hill) (1962).

[32] Analog computation in engineering design. Rogers \& Connolly (McGraw Hill Dunod) 1966.

[33] Analog simulation. Karplus (McGraw Hill) 1958.

[34] Analog computation. Fifer (McGraw Hill) 1961.

[35] Analog computers (SSSR). Eterman (Pergamon Press).

[36] Analog computing at ultra H.Speed. MacKay (Fisher-Wiley).

[37] Design fondamentals of analog computer components, Howe (D. Van Nostrand).

[38] Electronic analog and hybrid computers, Korn and Koin (McGraw Hill), 1964.

[39] Electronischer Analogrechner, Ernst, Oldenbourg (Munich).

[40] High speed analog computers, Tomovic, Karplus (Wiley and sons) 1962.

[41] Introduction to analog computation, Ashley (Wiley).

[42] Introduction to electronic analog computer, Warfield (Prentice-Hall).

[43] Analog method : computation and simulation, Karplus and Soroka (MacGraw Hill) 1959.

[44] Le calcul analogique par courants continus, DahlaixDumesnil (Dunod).

[45] Annales de l'association internationale pour le calcul analogique (1955-1958-1961-1964-1967-1970-1973). (Presses académiques européennes Bruxelles).

[46] Machines hybrides et leur application (1968), Laurent, Thèse Fac. Sci. Lille.

[47] Traitement des équations différentielles sur calculateurs électroniques, Karplus et Girerd (Gauthier Villars) 1968.

[48] Hybrid computation, Bekey, Karplus (John Willey and Sons) 1968.

[49] Calcul analogique et hybride, Stenberg (Masson) 1969. 